\title{
Ventro-Lateral Abdominal Hernia in Sheep and its Surgical Correction Techniques - A Case Report
}

\author{
Haben Fesseha ${ }^{1 *}$ and Friat Kidanemariam ${ }^{2}$ \\ ${ }^{1}$ School of Veterinary Medicine, Wolaita Sodo University, Ethiopia \\ ${ }^{2}$ College of Veterinary Science, Mekelle University, Ethiopia
}

\begin{abstract}
An Ewe of eighteen-month-old and weighing 37kg was presented to Mekelle University Veterinary Teaching Hospital Mekelle, Ethiopia with a swelling in the right ventrolateral abdominal wall for the last two weeks. Clinical examination reveals dull, depressed, reduced appetite and weakness and confirmed right-sided ventrolateral abdominal hernia for the last two weeks. The case was handled surgically by means of herniorrhaphy using overlapping mattress suture techniques and synthetic absorbable polyglycolic acid. The ewe was completely recovered on the 20th day postoperatively. Successful management of ventrolateral hernia in ewe is discussed.
\end{abstract}

KEYWORDS: Ewe; Herniorrhaphy; Overlapping mattress suture; Ventrolateral abdominal Hernia

\section{INTRODUCTION}

A hernia is defined as the protrusion or displacement of an organ, part of an organ, or tissue outside the abdominal cavity through an abnormal opening in the abdominal wall which can be noted from the outside of an animal's body and can be detected with an external examination. The opening is caused by a tear in the abdominal wall or it may be a natural opening like the inguinal canal or femoral canal. There are different types of hernia in small and large animals that are classified according to their anatomical locations into umbilical, inguinal, scrotal, femoral, perineal, and ventral (or abdominal) hernias [1,2].

A Ventral hernia or Ventro-lateral is defined as a hernia through any part of the abdominal wall other than a natural orifice and the hernia is ventral to the stifle skin fold $[1,3]$ or a ventral hernia is caused by the migration of viscera through a tear in the abdominal wall that is mostly due to trauma (kicks, horn thrust, violent contact with blunt object and sudden jump) that resulted in rupture of the prepubic tendon in large animals, particularly cow and mare. A ventral abdominal hernia is commonly found acquired condition in ruminants and horses and common in dog and pig compare to other domestic species $[2,4]$.

External Abdominal hernias are mostly caused due to a serious injury to the muscular portion of the abdominal wall. Thus, trauma such as kick by large animals (camel), horn thrust in cattle or violent contact with blunt objects or automobile accident or an abscess in the abdominal cavity and all these conditions may lead to weakening of the abdominal muscles lead to a ventral hernia. Besides, abdominal distension due to pregnancy or violent straining during parturition may lead to ventral hernia especially in sheep $[1,5]$. The hernial swelling varied in the location from the iliac crest to the lateral side of the thoracic cavity. These swellings did not correspond, with the size of their rings [6].

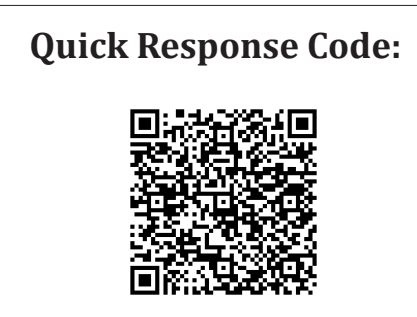

Address for correspondence: Haben Fesseha, School of Veterinary Medicine, Wolaita Sodo University, Ethiopia

Received: February 24, $2020 \quad$ Published: April 22, 2020

How to cite this article: Haben F, Friat K. Ventro-Lateral Abdominal Hernia in Sheep and its Surgical Correction Techniques - A Case Report. 2020 - 2(2) OAJBS.ID.000165. DOI: $10.38125 /$ OAJBS.000165 
The diagnosis of the hernia made through the contents of the hernia and hernia ring be clear or palpated the viscera under the skin, but the diagnosis of Irreducible hernias defaulted and needed to use the X-ray to reflect the lack of abdominal wall continuity [7]. Exploratory laparotomy used to diagnosis the defect, X-ray used to differentiate abdominal wall hernias from fibrin-cystic, abscess, and inflammatory swellings in bovine animals. Besides, such types of cases should be treated as soon as possible; otherwise, the prognosis will be grave $[1,8]$.

Herniorrhaphy is among the most performed operations in animal and human surgical practices. Most hernias enlarge over time and, if not repaired surgically, they may cause pain, anorexia, weight loss, or it may cause dystocia when a gravid horn is found in the hernial sac. Incarceration and strangulation of the bowel are the most dangerous life-threatening sequela of herniation [6,9]. Reports on hernias in small ruminants are very rare [4,10-12]. The present study describes the successful management of a ventral hernia in a sheep.

\section{CASE REPORT}

\section{History and Clinical Observations}

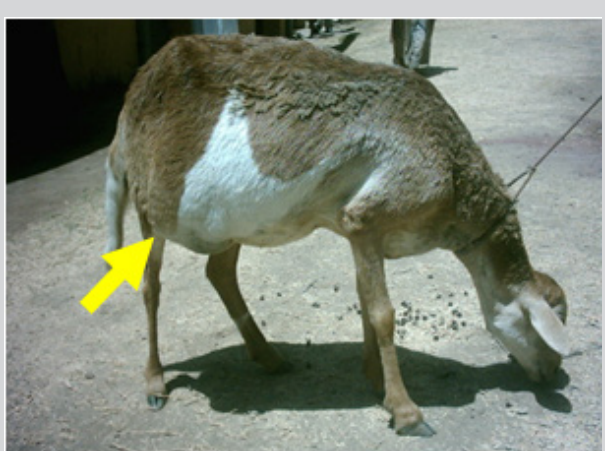

Figure 1: Ventrolateral hernia located lateral to the udder in Ewe.

An eighteen months old long-tailed Ewe weighing around $35 \mathrm{Kg}$ was presented to Veterinary Teaching Hospital, College of
Veterinary Science, Mekele University, with a history of swelling on the right side and lateral to the udder below the paralumbar fossa. However, increasing in size of the hernia was observed for the last two weeks and the owner does not know the exact cause of the case. On presentation, the ewe was dull, depressed with reduced appetite. The detail physical and clinical examination revealed reducible swelling near the right ventrolateral side of the Udder. The size of the hernial ring was 5 fingers in breadth (Figure 1). The clinical parameters viz. rectal temperature, heart rate, and respiratory rate were within normal range with good body condition. Based on clinical examination, the case was diagnosed as ventrolateral hernia and was corrected by herniorrhaphy (overlapping mattress sutures) using synthetic sterile absorbable polyglycolic acid.

\section{Preoperative Preparations and Anesthesia}

Food was withheld for 24 hours and water for 12 hours prior to surgery. Preoperatively the surgical site was prepared aseptically and kept in the Ventrolateral position. Ewe was sedated with an Intravenously administration of Sedil@ (Diazepam, Intas pharmaceutical Ltd., India) at a dose of $0.1 \mathrm{mg} / \mathrm{kg}$. Bodyweight. In addition, regional anesthesia was done through paravertebral nerve block was made by blocking T13, L1 and L2 nerves combined with topical infiltration using two percent lidocaine (Lidocaine hydrochloride $2 \%$, Jeil pharm. co. Ltd., Korea) with the dose rate 1 $\mathrm{ml} / \mathrm{cm}$ area and loaded in a syringe with 22-gauge, 1.5 -inch needle to desensitize the abdominal muscle and alleviate pain during surgical procedure.

\section{Surgical Correction and Treatment}

Following proper physical and chemical restraining and aseptic preparation of the surgical site, the Ewe was kept on appropriate direction for the next surgical procedure. A gently and enough longitudinal incision was done in the middle of the swelling. The skin was detached from the subcutaneous tissue through blunt dissection and the incision was continued through the abdominal muscle and peritoneum. Then all muscular layers together with skin were grasped with a handheld retractor to get enough surgical field and expose hernial ring (Figure 2).
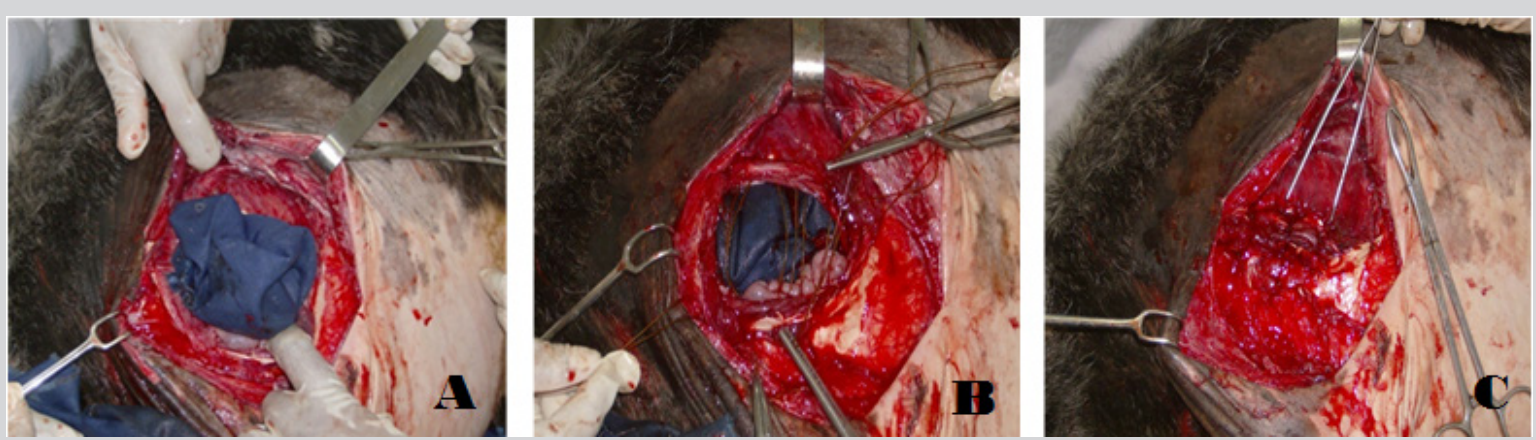

Figure 2: (A) Exposing the hernial ring and intestinal content after longitudinal incision; (B) Replacing the intestinal content into the abdominal cavity; (C) Overlapping mattress herniorrhaphy using synthetic sterile absorbable polyglycolic acid.

Upon insertion of a finger, slight adhesion of some part of the small intestine with the lower abdominal wall was found and it was gently detached, and the small intestine was were properly replaced to the abdominal cavity (Figure 2). Then, intentional elliptical incision was made on the edge of the hernial ring to facilitate the healing process and herniorrhaphy was done by overlapping mattress sutures using synthetic sterile absorbable polyglycolic acid (Shandong Sinorgmed Int'l Co., Ltd, China) suture materials size no. 2 (Figure 2). Moreover, excess skin of the sac was removed, and the subcutaneous tissue and skin were closed with an interrupted horizontal mattress using silk 2-0 size and the surgical site was properly cleaned and dressed in a $1 \%$ Povidone-iodine solution and admitted home. 


\section{Post-Operative Follow-Up and Result}

Postoperatively, Intermittent bleeding was noticed following skin suture, but it is normal and helps to clean the wound unless continued for a long period. The dressing of the wound was done using a $1 \%$ Povidone-iodine solution at second- and thirddays post-operative up to 14-days until it completely healed. Besides, the ewe was kept on fluid therapy (5\% Dextrose solution, $1000 \mathrm{ml}$ stat, I.V.), antibiotic Procaine penicillin $(24 \mathrm{mg} / \mathrm{kg})$ and dihydrostreptomycin sulphate $(30 \mathrm{mg} / \mathrm{kg}$ ) (Pen-Strep® Norbrook, Great Britain) was given intramuscularly for successive five days and Meloxicam $(0.5 \mathrm{mg} / \mathrm{kg}$, IM) for 3 successive days. Tetracycline wound spray was also applied around the wound. Furthermore, the owner was also advised to closely monitor the Ewe and advised to allow some exercise and supplied with good nutrition to facilitate wound healing. After 20-day follow-up post-operation, the wound was healed completely, and the Ewe was under good health status.

\section{DISCUSSION AND CONCLUSION}

Hernias have different congenital and acquired causes that have several deleterious effects, such as lowering the productivity and reproductivity of the affected animals. Ventral and Ventrolateral hernias are commonly seen in the ventral and ventrolateral abdominal wall near the midline and the size of the hernial opening varies in diameter and the nature of hernia contents depends on the site of the herniation $[1,13]$. It is a common condition; however, it is generally overlooked by most rural farmers unless it results in some serious symptoms $[6,10]$. In the case report, the exact cause of the hernia could not be traced, but it might be as a result of the weakening of the abdominal muscles due to violent trauma with blunt objects or any accidental injury. Nearly similar location was also noticed in the present study and there was a large hernial opening through which protruded the viscera.

According to the previous case reports, the incidence of ventral abdominal hernia was observed in sheep and goat different species and in different countries. There is a report in the ventral abdominal hernia of goat in 19 cases (32.2\%) in Pakistan. The cases are more common in females ( 42 cases) than in males (16 cases) and more common in animals above one year of age (16 cases) this is because of the fact that males are slaughtered regularly at a lifespan of 6 -12 months. Omentum and intestine are the most common contents (12 cases) and abomasum ( 3 cases), gravid uterus ( 2 cases) and rumen $(3$ cases) $[1,13]$. This finding was in line with the present case report with respect to sex and contents.

Most of the ventrolateral hernias were reducible with palpable rings. However, there are also some ventrolateral hernias that were irreducible with impalpable rings which were due to severe adhesions of the contents with the hernial rings and hernial sacs $[10,11]$. Similarly, In the current case report, the ventrolateral hernia was reducible with palpable rings however, there were slight adhesions of the intestinal content with the lower abdominal wall.

The diagnosis of a ventral abdominal hernia is easy as the hernial ring can be felt in most cases and prognosis is guarded. There are lots of treatment options for a ventral abdominal hernia that depends on the size of the hernial opening. The application of bandage, clamps or ligatures may be helpful in a few cases where the hernial ring is small. Surgical intervention (herniorrhaphy) is useful in case of the large hernial opening but in extensive ventral abdominal hernia may require herrnioplasty $[7,11]$. In the current report, the diagnostic and surgical approach agreed with the above reports.
The finding of different researchers showed that abdominal hernias were the most prevalent type of hernias in sheep and goats followed by umbilical hernias [10]. In another study in Egypt also revealed that higher incidence of different type of hernias were recorded in buffaloes (diaphragmatic hernia) followed by cattle (umbilical hernia), sheep and goats (ventral hernia), donkeys, and horses [7]. Similarly, In Nigeria, ovine species had the highest percentage of hernias followed by caprine and bovine species, ventral abdominal hernias were the most common type recorded [14]. This finding was in line with the current case report where ventral hernial is seen in Ewe.

In this case report, right side ventrolateral hernias were seen in adult multiparous Ewe. A similar finding was seen in previous reports by Roberts [5] where most ventrolateral were recorded in right. However, this contrasted with the report of Yasin [13] who reported that 12 left-sided ventrolateral hernias were recorded out of the 18 ventral abdominal hernias. This might be due to trauma, increased intra-abdominal pressure during pregnancy, loss of abdominal wall strength with age, and weakness of the abdominal muscles and tendinous support of the abdominal wall due to malnutrition causes ventral hernias $[10,13]$.

The flank and ventrolateral abdominal region in ruminants lack a continuous muscle covering, it has a rather narrow fleshy portion with holly tendinous nature and an extensive aponeurosis [15]. In different studies, for closing the hernial rings, different suture materials and patterns have been used. According to previous research outputs, absorbable suture materials like polyglycolic acid, polyglactin 910, or polydioxanone is recommended to close a different type of abdominal hernial ring by simple interrupted, simple continuous or interrupted cruciate patterns; tensionrelieving sutures such as near-far-far-near is used to close large abdominal wall defects $[8,16]$. Similarly, synthetic absorbable polyglycolic acid was used as suture material and no complication was observed without hernial recurrence. Accordingly, ventrolateral hernia can be managed through herniorrhaphy using overlapping mattress suture technique and synthetic absorbable polyglycolic acid as suture material and recommended as an alternative technique.

\section{REFERENCES}

1. Farman RH, SH Al-Husseiny, ANA Al-Ameer (2018) Surgical treatment of hernia in cattle: A review. Al-Qadisiyah Journal of Veterinary Medicine Sciences 17(2): 61-68.

2. Fossum TW (2013) Small animal surgery textbook. Elsevier Health Sciences.

3. Oehme FW (1988) Textbook of large animal surgery. Williams and Wilkins.

4. Jettennavar PS, Kalmath PG, Anil kumar MC (2010) Ventral abdominal hernia in a Goat. Veterinary World 3(2): 93.

5. Roberts SJ (1986) Veterinary obstetrics and genital diseases. Theriogenology.

6. Abdin-Bey M, Ramadan R (2001) Retrospective study of hernias in goats. Scientific Journal of King Faisal University (Basic and Applied Sciences) 2(1): 77-88.

7. Misk N, Misk T, Semieka M (2008) Hernias in some farm animals in $25^{\text {th }}$ World Buiatrics Congress.

8. Fubini SL, Ducharme N (2016) Farm animal surgery. Elsevier Health Sciences.

9. Slatter DH (2003) Textbook of small animal surgery. Elsevier Health Sciences. 
10. Al-Sobayil FA, Ahmed AF (2007) Surgical treatment for different forms of hernias in sheep and goats. J Vet Sci 8(2): 185-191.

11. Das B (2012) Successful management of ventral abdominal hernia in goat: a case report. International Journal of Natural Sciences 2(2): 60-62.

12. Sagar PV, Harish D, Babu PP (2010) Ventral hernia in an Ongole cow: A case report. Vet World 3(2): 90-91.

13. Yasin M (2017) Clinical observation and surgical management of external hernias in ruminants in Dohuk governorate. Bas J Vet Res 16(1): 349-368.
14. Tanko FS (2015) An eight-year retrospective study on the prevalence of hernias in large animals at the veterinary teaching hospital Ahmadu Bello University, Zaria. Animal and Veterinary Sciences 3(4): 125-127.

15. Dyce KM, Sack WO, Wensing CJG (2002) The ventrolateral wall of the abdomen, in Textbook of veterinary anatomy. Saunders, Philadelphia, USA.

16. Baxter GM (2004) Hernias/umbilicus in Farm animal surgery. In Fubini SL, Ducharme NM (Eds.) Saunders. pp. 477-483. 